\title{
The Nuclear Structure of 3C 84 with Space VLBI (RadioAstron) Observations
}

\author{
Gabriele Giovannini ${ }^{* 1,2}$, Monica Orienti ${ }^{2}$, Tuomas Savolainen ${ }^{3,4}$, Hiroshi Nagai ${ }^{5}$, \\ Marcello Giroletti ${ }^{2}$, Kazuhiro Hada ${ }^{2,5}$, Gabriele Bruni ${ }^{4}$, Jeffrey Hodgson ${ }^{4}$, Mareki \\ Honma $^{5}$, Motoki Kino ${ }^{6}$, Yuri Y. Kovalev' ${ }^{7}$, Thomas Krichbaum ${ }^{4}$, Sang-Sung Lee ${ }^{6}$, \\ Andrei Lobanov ${ }^{4}$, Bong Won Sohn ${ }^{6}$, Kirill Sokolovsky ${ }^{7}$, Peter Voitsik ${ }^{7}$, J. Anton \\ Zensus ${ }^{4}$. \\ ${ }^{1}$ Dipartimento di Fisica e Astronomia, Bologna University, Italy; ${ }^{2}$ Istituto di \\ Radioastronomia/INAF, Italy; ${ }^{3}$ Aalto University Metsähovi Radio Observatory, Finland. \\ ${ }^{4}$ Max-Planck-Institut für Radioastronomie, Germany; ${ }^{5}$ National Astronomical Observatory of \\ Japan, Japan; ${ }^{6}$ Korea Astronomy and Space Science Institute, Republic of Korea; ${ }^{7}$ Astro Space \\ Center of Lebedev Physical Institute, Russia; \\ E-mail: ggiovanneira.inaf.it
}

\begin{abstract}
The radio galaxy $3 \mathrm{C} 84$ is a representative of gamma-ray-bright misaligned active galactic nuclei (AGN) and one of the best laboratories to study the radio properties of subparsec scale jets. We discuss here the past and present activity of the nuclear region within the central $1 \mathrm{pc}$ and the properties of subparsec-sized components $\mathrm{C} 1, \mathrm{C} 2$ and $\mathrm{C} 3$. We compare these results with the high resolution space-VLBI image at $5 \mathrm{GHz}$ obtained with the RadioAstron satellite and we shortly discuss the possible correlation of radio emission with the gamma-ray emission.
\end{abstract}

12th European VLBI Network Symposium and Users Meeting,

7-10 October 2014

Cagliari, Italy

\footnotetext{
*Speaker.
} 


\section{Introduction}

The radio source 3C 84 is associated with the giant galaxy NGC $1275(\mathrm{z}=0.0176)$, which is the Brightest Cluster Galaxy (BCG) of the relaxed cooling flow cluster Abell 426 in Perseus. 3C 84 is known to be a bright radio source and has been studied extensively since the early days of radio astronomy. At low resolution the radio emission is extended and diffuse with a halo-core structure typical of BCG galaxies at the center of cooling clusters. In the central 10-pc scale region, there is a pair of symmetric lobes, with evidence of free-free absorption for the Northern one (Walker et al. 2000). These lobes were probably formed by the jet activity originating in the 1959 outburst (e.g., Asada et al. 2006). At higher angular resolution the bright central region is resolved into a bright core and a one-sided complex jet emission. According to Liuzzo et al. (2010), the one-sided emission is likely due to Doppler boosting effects of a highly relativistic jet at a small angle to our line-of-sight. The jet velocity decreases quickly because of the strong interaction between the jet and the cooling gas, giving rise to the symmetric structure visible in the central $10 \mathrm{pc}$ region. The radio flux has been monitored since 1960, and episodes of violent flux increase have been reported (Kellermann and Pauliny-Toth 1968; O’Dea et al. 1984). In the mid-1980s, the radio flux became exceptionally bright, more than $60 \mathrm{Jy}$ at centimetre wavelengths, and then it subsequently decreased to $\sim 10 \mathrm{Jy}$ by the early 2000s (Nagai et al. 2012). Around 2005, it was reported that the radio flux started to increase again (Abdo et al. 2009). Very Long Baseline Interferometry (VLBI) observations revealed that this flux density increase originated in the central pc-scale region and it was accompanied by an ejection of a new jet component (see the next Section).

NGC 1275 was detected in $\gamma$-rays by the Fermi Large Area Telescope (LAT) in August 2008 (Abdo et al. 2009). This detection is particularly noteworthy, because the source was not detected by the Energetic Gamma Ray Experiment Telescope (EGRET) of the Compton Gamma Ray Observatory (GRO) (Reimer et al. 2003). The new $\gamma$-ray flux density is about seven times higher than the upper limit from the EGRET observations. Intriguingly, the higher $\gamma$-ray emission coincides with the increase of the radio flux density.

In the next sections we will discuss the sub-parsec structure of this source and present a new high-resolution space-VLBI image obtained with RadioAstron. The redshift of $3 \mathrm{C} 84$ gives an angular-to-linear size conversion factor of $0.344 \mathrm{pc} / \mathrm{mas}$, assuming $\mathrm{H}_{0}=71 \mathrm{~km} \mathrm{~s}^{-1} \mathrm{Mpc}^{-1}, \Omega_{M}=$ 0.27 and $\Omega_{\Lambda}=0.73$. We assume a jet orientation with respect to the viewing angle to be $20^{\circ}-25^{\circ}$ (Abdo et al. 2009, Nagai et al. 2014, Tavecchio and Ghisellini 2014).

\section{Sub-parsec Radio Structure}

High resolution 43 and $86 \mathrm{GHz}$ VLBI images taken at the epochs before $\sim 2005$ (e.g. Dhawan et al. 1998), show a core (C1) and a nearby bright knot separated by 0.5 mas at a position angle $\sim 160^{\circ}$. Beyond 0.5 mas, there is an abrupt bend in the jet toward a position angle of $235^{\circ}$, where a diffuse component ( $C 2$ here, $\mathrm{D}$ in Dhawan et al. 1998) is present at a distance of 1.2 mas. In the first 4 epochs (from 2006/134 to 2007/258) of the multi-epoch monitoring presented by Nagai et al. (2010), the source structure is the same and similar to that seen in the $\gamma$-ray quiet phase. A clear change in the source structure is evident after the 2007/258 image and a new component C3 has emerged from the central core. 


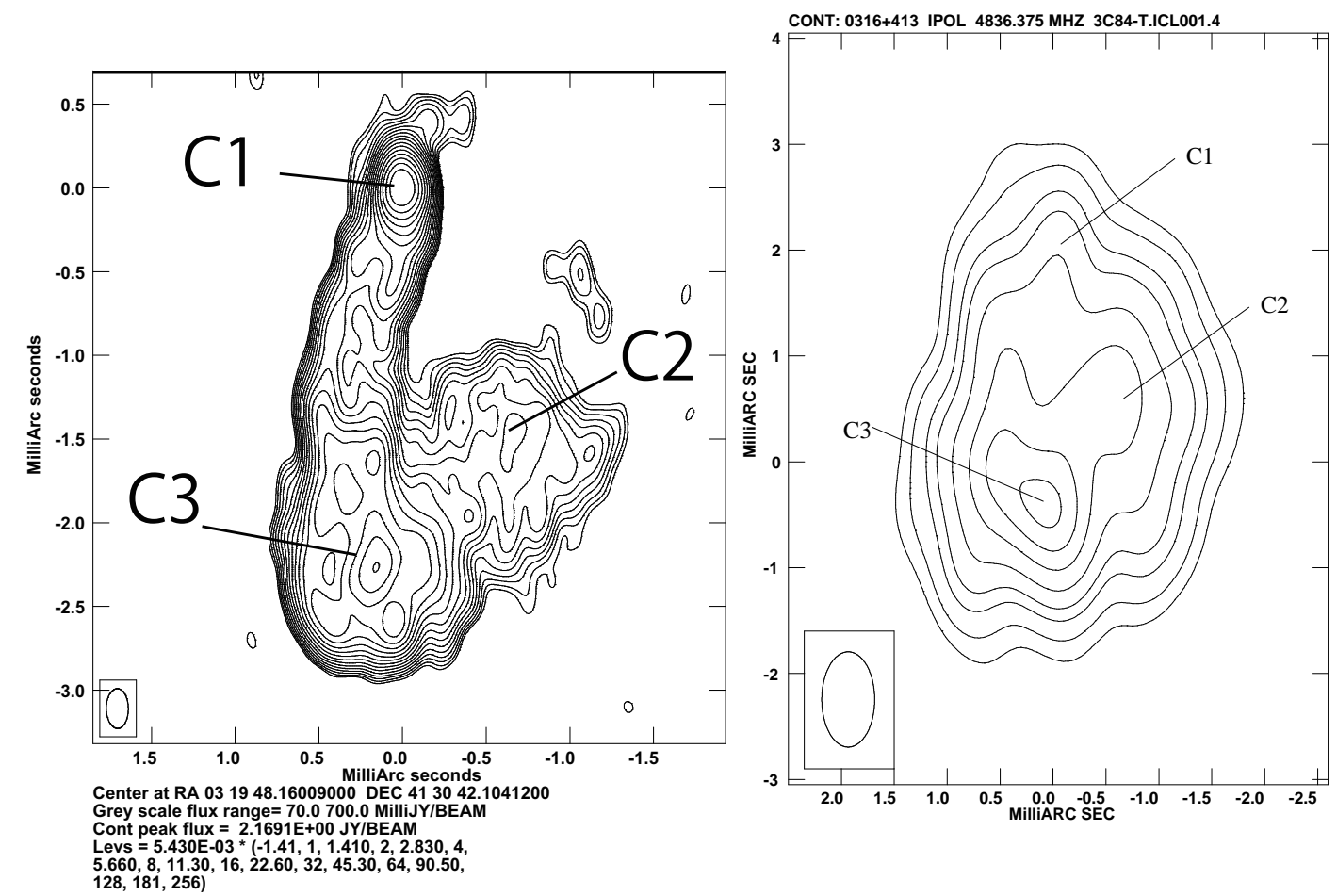

Figure 1: Left: $43 \mathrm{GHz}$ total intensity map of $3 \mathrm{C} 84$ obtained with the VLBA in January 2013. The HPBW is $0.24 \times 0.13$ mas in the PA of $0.7^{\circ}$. For more details see Nagai et al. (2014). Right: Space-VLBI image of $3 \mathrm{C} 84$ at $5 \mathrm{GHz}$. The HPBW is $0.9 \times 0.5$ mas. Countours are: 50, 100, 200, 300, 500, 800, 1100, and 1400 $\mathrm{mJy} /$ beam.

Using the VLBA archive data and monitoring observations with the VLBI Exploration of Radio Astrometry (VERA) array, Suzuki et al. (2012) found that this new component was ejected from the core after 2003 November 20th. The component C3 is advancing with an increasing apparent velocity (0.1c in November 2003, $0.47 \mathrm{c}$ in November 2008) toward a position angle of $\sim$ $170^{\circ}$. This direction is clearly unrelated to that of the component C2. Moreover, the jet PA and structure are quite different from the previous images. The flux density of $\mathrm{C} 3$ and $\mathrm{C} 1$ have clearly increased after 2008 and at $43 \mathrm{GHz}$ they showed about the same flux density (see Fig. 7 in Suzuki et al. 2012). After 2009 the core flux density at $15 \mathrm{GHz}$ has been almost constant while the flux density of $\mathrm{C} 3$ showed a further increase. The core flux density was $4.84 \mathrm{Jy}$ and the flux density of C3 was $9.85 \mathrm{Jy}$ in the MOJAVE $15 \mathrm{GHz}$ image on February 11, 2011 (see Lister et al. 2009 for the details of the MOJAVE project).

As discussed in Nagai et al. (2014), the relatively slow apparent velocity of C3 is at odds with the one-sidedness of the radio structure, the spectral energy distribution fit to the observed broadband spectrum, and with the one-zone synchrotron-self-Compton model. Therefore, we assume that the jet bulk motion is highly relativistic, while the observed slow proper motion is related to the jet advancing in a high density medium. In this interpretation $\mathrm{C} 3$ is a hot-spot like structure due to the interaction of the new jet structure with the external medium. 
At $43 \mathrm{GHz}$, the observations carried out using the VLBA on January 2013 (Nagai et al. 2014, Fig. 1) and by the Boston University's blazar monitoring program ${ }^{1}$ show an unresolved core (C1), a complex structure coincident with $\mathrm{C} 3$ and a diffuse emitting region $(\mathrm{C} 2)$. The jet connected to $\mathrm{C} 3$ is limb-brightened. We note that in the previous images (e.g., Dhawan et al. 1998, Romney et al. 1995, and Lister 2001) the jet activity was connected to the component C2, oriented at a different position angle and the jet profile was centrally peaked.

\section{Comparison of Radio and High Energy Emission}

Two $\gamma$-ray flaring periods have been reported: the first one in April-May 2009 (Kataoka et al. 2010) and the second one in June-August 2010 (Brown \& Adams 2011). During both of the $\gamma$-ray flares, components $\mathrm{C} 1$ and $\mathrm{C} 3$ showed a large change of the flux density, but on a time scale longer than that of the $\gamma$-ray activity. After 2010 the $15 \mathrm{GHz}$ flux density of $\mathrm{C} 3$ started to increase faster than that of $\mathrm{C} 1$, and at present $\mathrm{C} 3$ is the brightest sub-parsec feature at $15 \mathrm{GHz}$.

\section{RadioAstron Results}

3C 84 was observed with the 10-m Space Radio Telescope (SRT) of the RadioAstron Mission (Kardashev et al. 2012; 2013) at 5 and $22 \mathrm{GHz}$ as a part of the RadioAstron Nearby AGN Key Science Program (Savolainen et al., these proceedings). Observations were carried out on September 21, 2013, outside of the regular EVN sessions because of the SRT constrains. Science data from the SRT were transmitted in real-time to the telemetry stations in Puschino, Russia and Green Bank, USA, where they were recorded. The data presented here were correlated by using the space-VLBI version of the DiFX software (dra-DiFX, see Bruni et al. 2015) at the Bonn Astro/Geo correlator (MPIfR). This DiFX version allows to include a space-based antenna, given the reconstructed orbit, which for RadioAstron is provided by the ballistics group of the Keldysh Institute of Applied Mathematics of the Russian Academy of Sciences. Solutions for delay and delay-rate were searched for the SRT for all the scans. When no fringes were found, typically on the longest space baselines, delay and rate values extrapolated from previous scans were adopted in order to optimize the correlation window. RadioAstron fringes were clearly detected in postprocessing by both PIMA (Petrov et al. 2011) and AIPS softwares: the shortest baseline detection was between the SRT and Kalyazin (Kl) at $\sim 0.2$ Earth Diameters (ED), and the longest baseline with a clear detection $(8.9 \sigma)$ was between Effelsberg $(\mathrm{Eb})$ and the SRT, $~ 6.9 \mathrm{ED}$.

The ground array at $5 \mathrm{GHz}$ was: VLBA, 100-m Green Bank Telescope, Jodrell Bank, Onsala, Shanghai, Noto, Effelsberg, WSRT, Hartebeesthoek, and Kalyazin. We present here preliminary results at $5 \mathrm{GHz}$, while the $22 \mathrm{GHz}$ part of the experiment is still being processed.

The Jansky Very Large Array (VLA) observed as a phased array (Y27) mainly at $22 \mathrm{GHz}$, but during the times when RadioAstron was cooling its communications antenna, we used frequency agile ground telescopes, including Y27, to observe $3 \mathrm{C} 84$ also at 6,2 and $0.7 \mathrm{~cm}$. The observations with the phased VLA also provide the standard WIDAR correlation products and these were calibrated and imaged as well in order to obtain accurate total flux measurements. The array was in the

\footnotetext{
${ }^{1}$ http://www.bu.edu/blazars/VLBAproject.html
} 
$\mathrm{C} / \mathrm{B}$ configuration, and we measured the flux density of the unresolved core, obtaining $17.5 \mathrm{Jy}$ at $5 \mathrm{GHz}, 35.9 \mathrm{Jy}$ at $15 \mathrm{GHz}, 29.8 \mathrm{Jy}$ at $22 \mathrm{GHz}$, and $29.3 \mathrm{Jy}$ at $43 \mathrm{GHz}$. The radio spectrum is clearly absorbed at low frequencies with a peak around $15 \mathrm{GHz}$.

In the space-VLBI array the $(u, v)$ coverage is mostly in a narrow range of position angles around $\sim 100^{\circ}$. The poor, almost one-dimensional $(u, v)$ coverage on the space baselines makes it difficult to obtain images at full resolution, and modelling will be required in order to utilize the data on the longest baselines.

The VLBI data calibration mostly followed the standard procedures, except for a complicated bandbass calibration and scan-by-scan fringe fit with solution intervals varying as a function of expected residual acceleration of the SRT (see Savolainen et al., these proceedings). After a detailed editing of the data, we made images limiting the $(u, v)$ range to about $1 \mathrm{ED}\left(\sim 1.0 \times 10^{5} \mathrm{k} \lambda\right)$ and we self-calibrated the ground telescope gains in phase and amplitude. When a good image was derived we started to increase the $(u, v)$ range in imaging and self-calibration up to the maximum $(u, v)$ range $\sim 10^{6} \mathrm{k} \lambda$. We used a super-uniform weighting, which gives a low weight to short baselines, and we derived images at different resolutions using a combination of the $(u, v)$ range and weight.

In Fig. 1, we present a space-VLBI image with a HPBW of $0.9 \times 0.5$ mas $\left(\mathrm{PA}=0^{\circ}\right)$. In the image the nuclear component is weak due to (self-)absorbtion. The jet from the core to the component $\mathrm{C} 3$ is not resolved transversally. Component $\mathrm{C} 3$ is the brightest component within the central substructure and it is compact at $5 \mathrm{GHz}$. Component $\mathrm{C} 2$ shows a diffuse morphology.

We compared the VLBA data at $43 \mathrm{GHz}$ (at an epoch close in time, Aug. 2013) to RadioAstron data at $5 \mathrm{GHz}$ by making images with the same cell size and angular resolution and matching as much as possible the $(u, v)$ coverage. In the nuclear region and in the beginning of the jet, we have a spectral index $\alpha \sim-1.4\left(\mathrm{~S}(v) \propto v^{-\alpha}\right)$, i.e. these parts are strongly (self-)absorbed. The $\mathrm{C} 3$ component has a spectral index of $\alpha \sim-0.7$, also indicating self-absorption and thus confirming its compactness. The jet and $\mathrm{C} 2$ region show $\alpha \sim 0.2$ and 1.1 respectively.

\section{Conclusions}

From a comparison of the space-VLBI image of $3 \mathrm{C} 84$ at $5 \mathrm{GHz}$ to the available ground-based images, we can derive some preliminary results, while we are waiting for the higher resolution space-VLBI data at $22 \mathrm{GHz}$ :

1) The core (C1) is very compact and (self-)absorbed between 5 and $22 \mathrm{GHz}$.

2) The one-sided limb-brightened jet has on average a flat radio spectrum $(\sim 0.2)$.

3) The $\mathrm{C} 3$ component at the end of the jet, is compact and self-absorbed. At $15 \mathrm{GHz}$ it is the brightest component, and its brightness is still increasing with time. New space-VLBI data at higher resolution will give more information on this structure.

4) The $\mathrm{C} 2$ component is resolved with a steep spectrum confirming the interpretation that it is related to the previous activity, which has stopped well before the current multi-wavelength activity. 5) Properties of the previous ( $\gamma$-silent) activity period (related to component C2) and the present activity are quite different in the jet structure, its PA, and in the high energy activity.

The lack of a strong correlation between the $\gamma$-ray flares and the radio activity suggests that the origin of the high energy emission may be due to a 'spine-layer' scenario discussed by Tavecchio 
and Ghisellini (2014). However, the activity of the component C3, with an increasing flux density and a strong interaction with the external medium, could be another source of $\gamma$-ray emission.

Acknowledgements The RadioAstron project is led by the Astro Space Center of the Lebedev Physical Institute of the Russian Academy of Sciences and the Lavochkin Scientific and Production Association under a contract with the Russian Federal Space Agency, in collaboration with partner organizations in Russia and other countries. The National Radio Astronomy Observatory is a facility of the National Science Foundation operated under cooperative agreement by Associated Universities, Inc. The European VLBI Network is a joint facility of European, Chinese, South African and other radio astronomy institutes funded by their national research councils. This research is based on observations correlated at the Bonn Correlator, jointly operated by the Max Planck Institute für Radioastronomie (MPIfR), and the Federal Agency for Cartography and Geodesy (BKG). This research has made use of data from the MOJAVE database that is maintained by the MOJAVE team. TS was partly supported by the Academy of Finland project 274477.

\section{References}

Abdo, A.A., Ackermann, M., Ajello, M., et al.: 2009, ApJ, 699, 31

Asada, K., Kameno, s., Shen, Z.-Q., et al. 2006, PASJ, 58, 261

Brown, A.M., Adams, J.: 2011, MNRAS, 413, 2785

Bruni, G., et al.: 2015, PoS(EVN 2014)119

Dhawan, V., Kellermann, K.I., Romney, J.D.: 1998, ApJL 498, L111

Kardashev, N.S., Kovalev, Y.Y., Kellermann, K.I.: 2012, The Radio Sc Bull 343, 22 (arXiv:1303.5200)

Kardashev, N.S., Khartov, V.V., Abramov, V.V., et al.: 2013, ARep, 57, 153 (arXiv:1303.5013)

Kataoka, J., Stawarz, L., Cheung, C.C., et al., 2010, ApJ, 715, 554

Kellermann, K.I., and Pauliny-Toth, I.: 1968, AJ, 73, 874

Lister, M.L.: 2001, ApJ, 562, 208

Lister, M.L., Aller, H.D., Aller, M.F., et al.: 2009, AJ, 137, 3718

Liuzzo, E., Giovannini, G., Giroletti, M., Taylor, G.B.: 2010, A\&A, 516, 1

Nagai, H., Suzuki, K, Asada, K., et al.: 2010, PASJ 62, L11

Nagai, H., Orienti, M., Kino, M., et al.: 2012, MNRAS, 423, L122

Nagai, H., Haga, T., Giovannini, G., et al.: 2014, ApJ, 785, 53

O’Dea, C.P., Dent, W.A., Balonek: 1984, ApJ, 278, 89

Petrov, L., Kovalev, Y.Y., Fomalont, E.B., Gordon, D.: 2011, AJ, 142, 35

Reimer, O., Pohl, M., Sreekumar, P., Mattox, J.R.: 2003 ApJ, 588, 155

Romney, J.D., Benson, J.M., Dhawan, V., et al.: 1995, PNAS, 92, 11360

Suzuki, K., Nagai, H., Kino, M., et al.: 2012, ApJ, 746, 140

Tavecchio, F., Ghisellini, G.: 2014, MNRAS, 443, 1224

Walker, R. C., Dhawan, V., Romney, J. D., et al. 2000, ApJ 530, 233 